\title{
Neuroleptic Malignant Syndrome Developing after Acute Overdose with Olanzapine and Chlorpromazine
}

\author{
Enasio Morris, MB BSa , Digby Green, BSc, MB ChBa ${ }^{a}$ Andis Graudins $M B B S, P h D^{a, b}$
}

aPrince of Wales Clinical School, Faculty of Medicine, University of New South Wales, Kensington, Australia

bClinical and Experimental Toxicology Unit, Department of Emergency Medicine, Prince of Wales Hospital, Randwick, Australia

\begin{abstract}
Neuroleptic malignant syndrome (NMS) is a relatively uncommon side effect that may develop after a recent increase in the therapeutic dose of an antipsychotic medication or the addition of a new agent in therapeutic doses.

Case Report: We report a case of NMS developing in a 36-year-old female patient 2 days following deliberate self-poisoning with $30 \times 10$-mg olanzapine tablets, $7 \times 100$-mg chlorpromazine tablets and an unknown amount of escitalopram. These were the patient's own medications. She had not been taking these for several weeks. The patient initially presented with sedation from her overdose which resolved over the next 24 hours. Following this, over the subsequent 24 hours, she became progressively confused, ataxic, hypertonic, ferbrile and tachycardic, with marked lead pipe rigidity of the limbs. Head CT, lumbar puncture and septic screen were all negative. She was treated with intravenous midazolam infusion, nasogastrically administered bromocriptine, external cooling and was mechanically ventilated. She gradually improved over a period of 10 days, with residual confusion lasting another week, and was discharged well with no deterioration from her premorbid neurologic state.

Conclusion: To our knowledge, although there are numerous cases reported with therapeutic use, NMS has not been reported to develop following acute olanzapine overdose. Clinicians should be aware that this may be an uncommon side effect of antipsychotic medication.
\end{abstract}

\section{INTRODUCTION}

Neuroleptic malignant syndrome (NMS) is an uncommon toxic syndrome commonly associated with recent commencement or increase in therapeutic dose of antipsychotic medications. It is suspected when a constellation of autonomic instability and neuromuscular and central nervous system (CNS) abnormalities develop over time. Clinical features may include gradual onset of pyrexia, tachycardia, lead-pipe muscle rigidity and hypertonicity, altered mental status, leukocytosis, and elevated serum creatinine phosphokinase. Olanzapine is a relatively new atypical antipsychotic agent that has a lower affinity for dopaminergic receptors than older antipsychotic drugs and also binds antagonistically to serotonin receptors. These characteristics result in fewer extrapyramidal side effects compared to older antipsychotic agents. Olanzapine has been used increasingly in the treatment of psychosis due to purported lower incidence of side effects than seen with classical neuroleptics. However, numerous case reports describing NMS have been reported with therapeutic use of this agent [1-5]. In previous cases, NMS has been observed in patients on therapeutic doses of other antipsychotic agents, when olanzapine subsequently had been added, or when it had been started as a new single therapeutic agent. Acute overdose with olanzapine results in progressive dose-dependent sedation, sinus tachycardia, anticholinergiclike delirium, agitation, and coma [6]. To our knowledge, we are unaware of any reports of overdose with newer, atypical antipsychotics resulting in subsequent NMS. Cohen et al. report a case of olanzapine overdose in a 31-year-old

Keywords: neuroleptic malignant syndrome, olanzapine, chlorpromazine, deliberate self-poisoning, drug toxicity Notes: There was no outside funding of any kind used for this study.

Corresponding Author: Andis Graudins, MB BS, Department of Emergency Medicine, Prince of Wales Hospital, Barker Street, Randwick, NSW, 2031, Australia.Email: Andis.graudins@unsw.edu.au 
female with agitation, coma, and anticholinergic signs, necessitating endotracheal intubation and sedation. The patient became extremely agitated and required physical restraint while intubated. She was extubated 26 hours postoverdose and complained of sore throat, muscle pains, and fever for the next 24 hours, with a peak creatine kinase level of 3,108 U/L [7]. The authors made no mention of the presence of muscle rigidity, hyperreflexia, or other neuromuscular features of NMS. They theorized that the patient may have exhibited signs of mild NMS or, alternatively, the symptoms may have been the result of anticholinergic delirium and agitation [7]. We report a case of severe NMS developing 24 hours following initial recovery from acute olanzapine and chlorpromazine self-poisoning.

\section{CASE REPORT}

A 36-year-old woman with a background history of schizophrenia presented to the emergency department (ED) 4 hours postingestion of thirty 10-mg olanzapine tablets, seven 100-mg chlorpromazine tablets, and an unknown quantity of escitalopram. On arrival she was noted to be drowsy, with a Glasgow Coma Score of 13. Her heart rate was 100 beats/min, respiratory rate 20 breaths per minute, she was afebrile, and blood pressure was 110/70 $\mathrm{mmHg}$. Neurological exam was nonfocal, with normal muscle tone and reflexes. The patient was drowsy but responding to vocal commands and had an intact gag reflex. Twelve-lead ECG showed sinus rhythm with a rate of $100 \mathrm{bpm}$ and normal QRS and QT intervals. She was admitted for cardiorespiratory monitoring. Her mental state did not deteriorate overnight and she did not require endotracheal intubation, but subsequent chest radiograph revealed evidence of probable right midzone aspiration pneumonia 12 hours postadmission. Intravenous antibiotics were commenced at this time. However, her mental state continued to improve over the next 24 hours and the patient was awake, alert, fully oriented, and ambulant with normal vital signs and clearing of her respiratory signs on day 2 postingestion. On interrogation, she confirmed the history and doses of olanzapine and chlorpromazine ingested, and also stated that she had not been taking her olanzapine in the several weeks prior to her presentation. The patient was transferred to a general medical ward 48 hours postingestion, awaiting psychiatric assessment. The following day, day 3 postingestion, she was noted to be mute, confused, not obeying commands, and incontinent of urine and feces. Her heart rate was $110-120$ beats/min sinus rhythm, blood pressure of $140 / 80$, temperature of $37.8^{\circ} \mathrm{C}$. Neurological examination revealed generalized hyperreflexia, with normal tone and no evidence of clonus or myoclonus, fasciculations or nystagmus. Her gait was ataxic, stooped, wide-based, and shuffling. Investigations at this time included a normal cranial CT scan and lumbar puncture, a serum white blood cell count of 28,900, serum creatinine $216 \mu \mathrm{mol} / \mathrm{L}$ (previously $86 \mu \mathrm{mol} / \mathrm{L}$ on admission, normal range 50-110), normal electrolytes and a creatine kinase of 200 IU/L. At this stage, a diagnosis of probable developing neuroleptic malignant syndrome was made and she was readmitted to the intensive care unit (ICU).
A nasogastric tube was inserted and bromocriptine commenced at $2.5 \mathrm{mg}$ qid, and increased to $5 \mathrm{mg}$ every 4 hours over the next 12 hours. By day 4 postingestion, the patient had deteriorated neurologically with increasing temperature up to $38.9^{\circ} \mathrm{C}$, increasing cogwheel muscle tone with lead-pipe rigidity. A midazolam infusion was commenced and temperature successfully controlled with an external cooling blanket. By day 6 , the patient's mental state continued to deteriorate, with poor ventilatory effort and no improvement in her neuromuscular signs despite bromocriptine therapy. She was electively intubated for respiratory support. Electroencephalogram showed slow wave activity consistent with chemical sedation. Creatine kinase levels peaked at $2260 \mu \mathrm{mol} / \mathrm{L}$ on day 6 , as shown in Table 1.

Given the prolonged course of her symptoms and lack of obvious response to bromocriptine by day 7 , the neuropsychiatric team was consulted with regards to possible treatment with electroconvulsive therapy (ECT). However, muscle tone and mental state began to improve over the next 24 hours, obviating the need for ECT. Serial serum procalcitonin levels were consistently less than $0.5 \mathrm{ng} / \mathrm{ml}$, indicating that bacterial infection was an unlikely cause of her ongoing fever. Blood cultures did not reveal any pathogenic bacteria. By day 8 , she was responding to voice with marked improvement in her muscle tone. She remained normothermic without external cooling. Renal function had returned to normal and CK was $254 \mathrm{IU} / \mathrm{L}$. At days 10 to 13 postoverdose, she was weaned off bromocriptine as she continued to improve. She was extubated on day 11. Residual confusion and short-term memory deficits persisted for another week, but the patient recovered completely by the third week post-overdose and was discharged with no deterioration from her premorbid normal level of functioning.

\section{DISCUSSION}

NMS is an idiosyncratic, life-threatening reaction usually resulting from the escalation of therapeutic doses of antipsychotics. It is thought to be caused by the blockade of dopamine receptors (D2) in the nigro-striatal pathway [8,9]. The incidence of NMS in patients chronically treated with neuroleptic agents has been reported as between $0.07 \%$ and $0.9 \%[8,9]$. We report a notable case of NMS developing after recovery from the acute sedative toxicity resulting from deliberate self-poisoning with olanzapine and chlorpromazine.

Olanzapine has a high affinity for serotonin receptors (5HT2a/2c), dopamine D1-4, muscarinic M1-6 histamine (H1) and adrenergic $\alpha-1$ receptors $[6,10]$. Since olanzapine is a weaker dopamine receptor antagonist than the traditional neuroleptics, it is thought to have less risk of extrapyramidal side effects and NMS, but there has not been any prospective evaluation performed to confirm these assertions [11]. Additionally, as our patient also ingested chlorpromazine-an older, typical antipsychotic agent with less specific antagonistic effects at dopamine receptors-it is likely that the combined antagonism of dopaminergic receptors by these 2 drugs compounded the risk of development of NMS. 


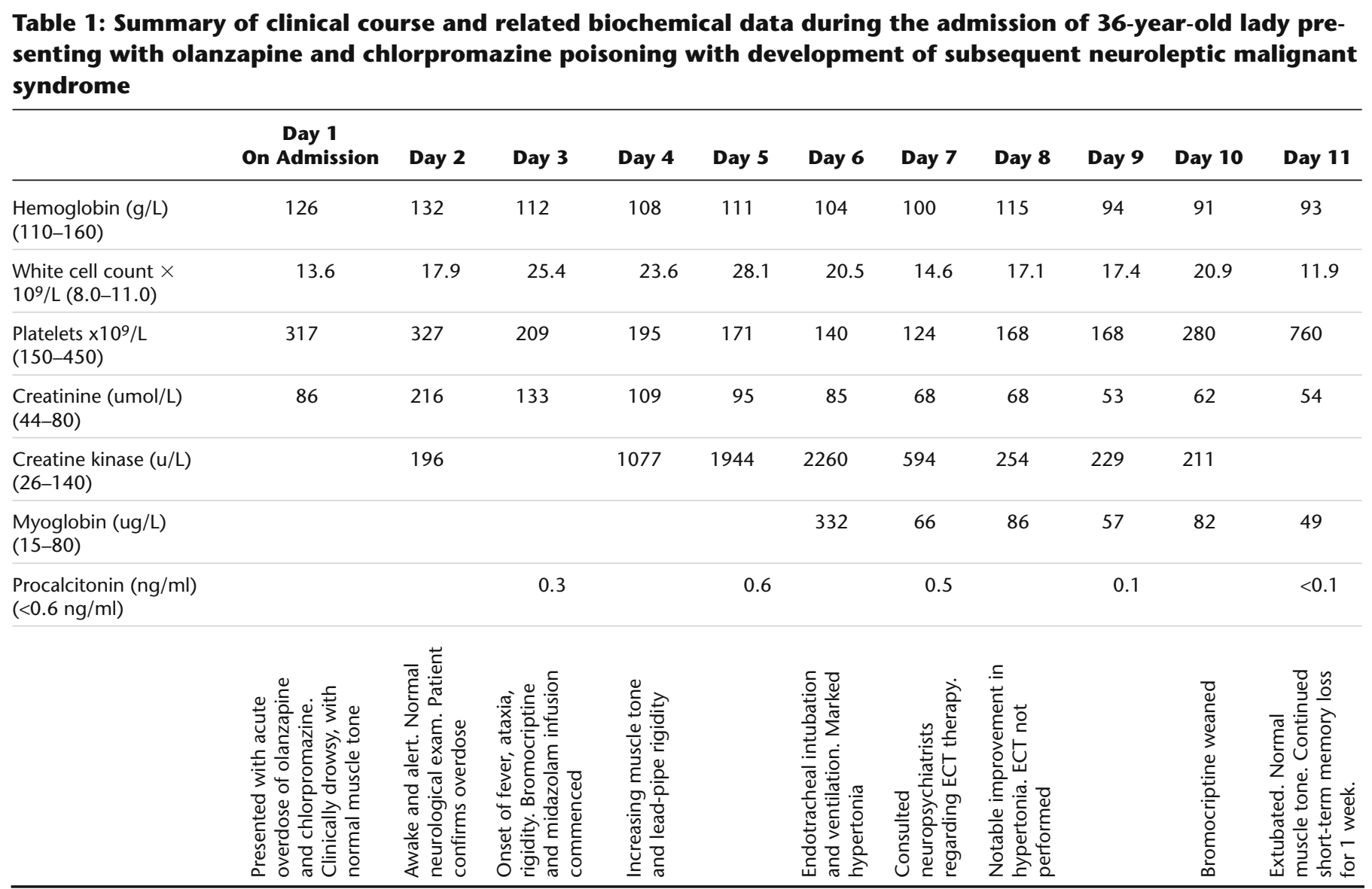

Several cases of NMS associated with therapeutic olanzapine dosing are reported in the literature $[5,10]$. These cases were associated with either a recent increase in the therapeutic dose of olanzapine or the addition of or a change from another antipsychotic agent $[4,11]$. Features of NMS in cases following therapeutic treatment with olanzapine and other atypical antipsychotics commonly follow a typical pattern with autonomic instability, hypertonia and lead-pipe rigidity, and mental status changes $[12,13]$. However, there have been no case reports of NMS documented in the literature following overdose of olanzapine or overdose of olanzapine and chlorpromazine. A Medline and Embase search combining the terms olanzapine, antipsychotic agents, overdose, poisoning, self-harm, suicide attempted, and neuroleptic malignant syndrome did not find any reports of NMS resulting from acute overdose of this atypical antipsychotic agent.

Olanzapine overdose commonly results in dose-dependent sedation and coma. It may also result in significant anticholinergic toxicity with sinus tachycardia, agitation, confusion, and delirium. Anticholinergic effects may be seen acutely or during the recovery phase of toxicity [6]. Symptoms commonly resolve over hours to days depending on the size of the overdose and do not usually result in any long-term sequelae. Once the acute sedative toxicity resolved in our patient, the clinical features that developed subsequently satisfied the diagnostic criteria for NMS established by DSM-IV and several other authors $[14,15]$. She took her neuroleptic overdose after a break of several weeks with the addition of a small amount of a second agent. There was a gradual deterioration in her mental state, with pyrexia, tachycardia, hypertonicity with lead-pipe rigidity, and elevated creatinine kinase. Symptoms developed 2 days post-ingestion, after her mental state had improved to normal from the initial drug intoxication. Although the patient also ingested a small amount of escitalopram, her clinical signs were not consistent with serotonin syndrome. This syndrome is commonly seen earlier after overdose and does not have the gradual onset or protracted duration of NMS. Neuromuscular features of serotonin syndrome are also different from those seen in NMS, with a preponderance of hyperreflexia, myoclonus, and inducible clonus being more prominent as neuromuscular manifestations of the syndrome [16]. It is also unlikely that our patient's clinical deterioration could be explained by a further overdose in hospital as she was constantly supervised by a nurse while in the ICU and on the general medical ward. Other differential diagnoses include possible alcohol or sedative hypnotic withdrawal, which can manifest with autonomic instability and mental status changes, delirium, and seizures. The neuromuscular signs and time course of the onset of illness postoverdose seen in this patient were not consistent with these diagnoses. Finally, neurosepsis may also manifest with fever, autonomic 
changes, and mental status changes. In our patient, lumbar puncture was normal, blood cultures were negative, and serial procalcitonin estimations were normal, suggesting that sepsis was an unlikely explanation for the symptom complex described here.

The management of patients with NMS includes admission to the ICU with attention to good supportive care, fluid management and electrolyte balance, maintenance of normothermia, sedation, and respiratory support. The gradual improvement in mortality from NMS in recent years is most likely attributable to greater attention to supportive care and early admission to the ICU for ongoing management rather than the use of any specific antidotal or antagonist agents. Specific chemical agents such as bromocriptine and amantadine-both being dopamine agonists—may have a role in the treatment of this syndrome, but there are currently no randomized controlled studies to confirm their efficacy. Case reports and observational studies suggest that bromocriptine may reduce the severity and duration of neuromuscular signs in NMS, but the response to this agent has never been assessed in a randomized, controlled study [17-19]. Dantrolene, a peripherally active muscle relaxant, has also been used in treatment in NMS based on its efficacy in malignant hyperthermia. Unlike malignant hyperthermia, in which rigidity and hyperthermia are the result of abnormal sequestration of calcium in the sarcoplasmic reticulum of skeletal muscle, the muscle rigidity and hypertonia seen in NMS is the result of CNS toxicity. There is no evidence to suggest that dantrolene is more effective than neuromuscular blockade and/or sedation in relief of NMS-induced muscle rigidity. We elected not to use this agent or to paralyze our patient with neuromuscular blockers, as her hyperthermia was well controlled with external cooling and intravenous midazolam infusion. Additionally, our patient's serum creatine kinase levels were not precipitously elevated. Electroconvulsive therapy (ECT) has also been reported to be effective in reversal of signs of NMS toxicity in refractory or prolonged cases of muscle rigidity. Various case reports have suggested that ECT might be an effective and life-saving therapy in patients with severe drug resistant NMS, and therefore should be strongly considered in such a situation $[20,21]$. ECT was initially considered in our patient after no improvement in her clinical condition after 7 days, but her mental state and muscle rigidity was notably better the following day, and we did not proceed with this therapeutic option.

When our patient recovered from her acute illness, she had residual memory loss and confusion for a week. Persistent shortterm memory loss has been previously reported post-NMS and has persisted for up to 10 months in some patients [22].

In conclusion, we report a case of significant NMS developing after the recovery from symptoms and signs of acute overdose with olanzapine and chlorpromazine. The patient improved to her premorbid state with supportive care and bromocriptine therapy. Clinicians should be aware that NMS may be an uncommon complication of atypical antipsychotic overdose, particularly if more than one antipsychotic agent has been ingested.

The authors have no potential financial conflicts of interest to report.

\section{REFERENCES}

1. Goveas JS, Hermida A. Olanzapine induced typical neuroleptic malignant syndrome. J Clin Psychopharmacol 2003; 23(1):101-102.

2. Nielsen J, Bruhn AM. Atypical neuroleptic malignant syndrome caused by olanzapine. Acta Psychiatr Scand 2005;112(2): 238-240.

3. Hall KL, Taylor WH, Ware MR. Neuroleptic malignant syndrome due to olanzapine. Psychopharmacol Bull 2001; 35(3):49-54.

4. Kontaxakis VP, Havaki-Kontaxaki BJ, Christodoulou NG, et al. Olanzapine-associated neuroleptic malignant syndrome. Prog Neuropsychopharmacol Biol Psychiatry 2002; 26(5):897-902.

5. Sierra-Biddle D, Herran A, Diez-Aja S, et al. Neuroleptic malignant syndrome and olanzapine. J Clin Psychopharmacol 2000;20(6):704-705.

6. Morgan M, Hackett LP, Isbister GK. Olanzapine overdose: A series of analytically confirmed cases. Int Clin Psychopharmacol 2007;22(3):183-186.

7. Cohen LG, Fatalo A, Thompson BT, et al. Olanzapine overdose with serum concentrations. Ann Emerg Med 1999; 34(2):275-278.

8. Goveas JS, Hermida A. Olanzapine induced "typical" neuroleptic malignant syndrome. J Clin Psychopharmacol 2003; 23(1):101-102.

9. Nielsen J, Bruhn AM. Atypical neuroleptic malignant syndrome caused by olanzapine. Acta Psychiatr Scand 2005;112(3): 238-240; discussion 40.

10. Philibert RA, Adam LA, Frank FM, et al. Olanzapine usage associated with neuroleptic malignant syndrome. Psychosomatics 2001;42(6):528-529.

11. Gelenberg AJ, Bellinghausen B, Wojcik JD, et al. A prospective survey of neuroleptic malignant syndrome in a short term psychiatry hospital. Am J Psychiatry 1988;145: 517-518.

12. Hansan S, Buckley P. Novel antipsychotics and the neuroleptic malignant syndrome. Am J Psychiatry 1998;155(8): 1113-1116.

13. Kontaxakis VP, Havaki-Kontaxaki BJ, Christodoulou NG, et al. Olanzapine-associated neuroleptic malignant syndrome: Is there an overlap with the serotonin syndrome? Ann Gen Hosp Psychiatry 2003;2(1):10.

14. Pope HGJ, Keck PEJ, McElroy SL. Frequency and presentation of neuroleptic malignant syndrome in large psychiatric hospital. Am J Psychiatry 1986;143:1227-1233.

15. Sachdev P, Mason C, Hadzi-Pavlovic D. Case control study of neuroleptic malignant syndrome. Am J Psychiatry 1997; 154:1156-1158.

16. Dunkley EJ, Isbister GK, Sibbritt D, et al. The Hunter Serotonin Toxicity Criteria: simple and accurate diagnostic decision rules for serotonin toxicity. QJM 2003;96: 635-642. 
17. Keck PE Jr, Pope HG Jr, McElroy SL. Frequency and presentation of neuroleptic malignant syndrome: a prospective study. Am J Psychiatry 1987;144:1344-1346.

18. Stanfield SC, Privette T. Neuroleptic malignant syndrome associated with olanzapine therapy: a case report. J Emerg Med 2000;19:355-357.

19. Apple JE, Van Hauer G. Neuroleptic malignant syndrome associated with olanzapine therapy. Psychosomatics 1999;40(3): 267-277.
20. Ozer F, Meral H, Ayadin B, et al. Electroconvulsive therapy in drug-induced psychiatric states and neuroleptic malignant syndrome. J ECT 2005;21(2):125-127.

21. Scheftner WA, Shulman RB. Treatment choice in neuroleptic malignant syndrome. Convuls Ther 1992;8(4): 267-279.

22. Rotheke S, Bush D. Neuropsychological sequelae of Neuroleptic Malignant Syndrome. Biol Psychiatry 1986;21: 838-841.

\section{BRIEF TOXICOLOGY COMMUNICATIONS}

\section{What Is the Origin of These Nail Changes in an Otherwise Healthy Young Patient?}

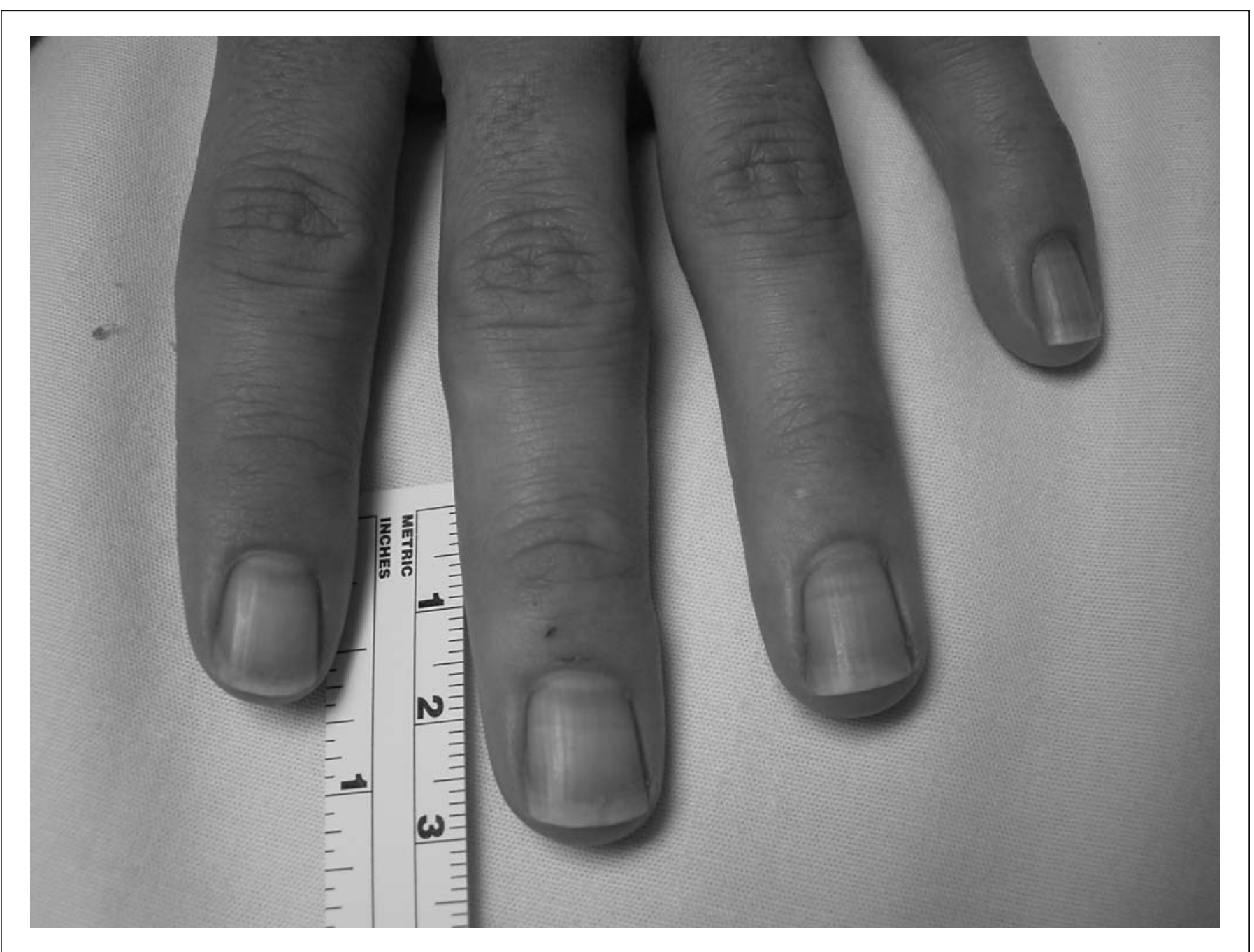

For the answer, see page 39. 